\title{
Inhibitory control over no-longer-relevant information: Adult age differences
}

\author{
LYNN HASHER, MARY BETH QUIG, and CYNTHIA P. MAY \\ Duke University, Durham, North Carolina
}

\begin{abstract}
Hartman and Hasher (1991) used a garden-path task in which younger and older adults generated the final word for each of a series of high-cloze sentences. Under instructions to remember the final word, the experiment included critical sentences for which the generated word was replaced by a new, to-be-remembered target. Using an implicit priming task, the first experiment replicated a basic finding: Younger adults showed priming only for the target words, whereas older adults showed priming for both the generated and target words. Two experiments explored boundary conditions. One showed that an additional sentence that interpreted the new target word enabled older adults to narrow access to only the target word. The provision of additional time following the introduction of the new target word did not. Specific information, not more time, is required for inefficient inhibitory mechanisms to clear the recent past from memory.
\end{abstract}

One model of cognition proposes that, together with goals, inhibitory attentional mechanisms control at least three aspects of the contents of consciousness and of action: (1) they determine which activated representations gain entrance into working memory, (2) they suppress those representations in working memory that are no longer relevant, and (3) they help to prevent prepotent or recently rejected candidates for response from gaining control over thought and action before weaker alternative candidates can be considered (Hasher, 1994; Hasher \& Zacks, 1988; see also Stoltzfus, Hasher, \& Zacks, 1996; Zacks, 1995; Zacks \& Hasher, 1994). Applied to language comprehension, attentional inhibition thus functions to keep processing efficient by maintaining activation centered along the intended meaning of a message (see Stoltzfus, Hasher, Zacks, Ulivi, \& Goldstein, 1993; see also Gernsbacher, 1990; Kintsch, 1993).

When inhibitory mechanisms are inefficient, a broader range of information will enter working memory, information that is no longer relevant will continue to remain active, and the frequency of inappropriate overt responses and of momentary, irrelevant or marginally relevant thoughts will increase. Under what conditions and for whom is inhibition likely to be inefficient? Recent work strongly suggests that inhibition is less efficient at non-

This research was supported by Grant AGO 4306 from the National Institute on Aging. Portions of this manuscript were prepared with the help of a grant from the James McKeen Cattell Foundation and with the support of the Department of Psychology, University of Toronto, and the Rotman Research Centre of Baycrest Hospital, North York, Ontario. The authors are particularly grateful to Marilyn Hartman, who was extremely helpful in the initial stages of this project, and to Michael $J$. Kane for his invaluable contributions to this project. C. P. May is now at the Department of Psychology, University of Arizona. Correspondence should be addressed to L. Hasher, Department of Psychology, Box 90085, Duke University, Durham, NC 27708 (e-mail: Ihasher@acpub.duke.edu). optimal times of the day than at optimal times of day (May, 1995; May \& Hasher, in press). Inhibition may also be less efficient for depressed and stressed young adults relative to age-mate controls (Linville, 1996), as it may be for younger children relative to older children (e.g., Dempster, 1992; Harnishfeger \& Bjorklund, 1993; Pennington, 1994), for people with schizophrenia (e.g., Beech \& Claridge, 1987; Frith, 1979), with frontal lobe damage (Shimamura, 1995), with attention-deficit disorder (Pennington, Groisser, \& Welsh, 1993), and with obsessivecompulsive disorder (Ferraro, Johnson, \& Wonderlich, 1995).

Inhibition is also considerably less efficient for older adults than for younger adults (e.g., Dempster, 1992; McDowd, Oseas-Kreger, \& Filion, 1995; Stoltzfus et al., 1996; Zacks \& Hasher, 1994). For example, older adults have a broader range of information active in working memory than do younger adults (Hamm \& Hasher, 1992; Stoltzfus, 1992; see also Laver, 1992, 1994). In reading, older adults are likely to form multiple inferences in situations in which younger adults do not. For example, older adults infer that multiple instruments (e.g., both a camera and a gun) are consistent with a scenario that includes a person on a photographic safari who is about to take a "shot" (Hamm \& Hasher, 1992). By contrast, younger adults, exposed to the same information, infer a single instrument (for this example, a camera). Similarly, older adults have speeded access both to a normatively predicted word as the ending of a high-cloze sentence (e.g., tenants, for the sentence "The landlord was afraid of a strike by his___, and to a normatively unlikely ending of that same sentence (e.g., residents; Stoltzfus, 1992). By contrast, younger adults have speeded access only to the normatively predicted ending (tenants). Thus, diminished inhibition appears to result in a broader range of information entering working memory for older adults, enriching its contents relative to that of younger adults. 
The working memories of older adults are additionally enriched because, relative to younger adults, they are less able to abandon no-longer-relevant thoughts and action plans from working memory (Zacks \& Hasher, 1994). The need to clear no-longer-relevant information from working memory is critical across domains of behavior. In comprehension, for example, topics, settings, and focal characters all change with regularity in both spoken and written discourse. The failure to efficiently abandon nolonger-relevant topics, for example, can easily result in disruptions to understanding. Similarly, the need to clear working memory also occurs when an initial interpretation is incorrect, as occurs when there has been a misunderstanding. Such misunderstandings are often simulated in the laboratory by using "garden-path" sentences and passages. These are materials in which an initial interpretation proves to be incorrect, requiring a new interpretation for a final, accurate understanding of the message. Younger adults have relatively little difficulty with garden-path materials. When reading, they respond immediately to the critical information that disconfirms their original understanding, and they quickly generate a new, more appropriate understanding (Zacks \& Hasher, 1988). Having done this, younger adults abandon their first, nolonger-correct interpretation (Hamm \& Hasher, 1992). Although older adults also both respond immediately to disconfirming information and generate an appropriate new understanding (Stine, Ruh, \& Hindman, 1994), they do not abandon their initial interpretation (Hamm \& Hasher, 1992).

A garden-path sentence-processing task introduced by Hartman and Hasher (1991) also assesses the impact of inhibition on deleting no-longer-relevant ideas from working memory. In this task, participants read and then generate a final word for each of a series of high-cloze sentence frames (e.g., stamp for "He mailed the letter without a "). For critical sentences, the generated word is replaced by a new word (e.g., check). For noncritical sentences, the generated word is not replaced. For all sentences in the series, participants are instructed to remember the final word. An indirect test of the accessibility of the two alternative endings to critical sentences, the self-generated but disconfirmed one (stamp) and the to-be-remembered target (check), is given subsequently in the guise of collecting norms for medium-cloze sentence frames, each of which is missing its final word. So, for the two endings of the example critical sentence above, there are two medium-cloze sentences that are given to participants on the subsequent indirect test: "For his collection he found a particularly rare " ( for $s t a m p)$, and "She paid all her bills by ___ (for check). The measure of sustained access to either or both of the endings for each critical high-cloze sentence is defined as priming above-baseline completion rates (approximately $50 \%$ for naive participants) for each medium-cloze sentence. Consistent with the Hamm and Hasher (1992) work suggesting that younger adults are efficient at clearing no-longer-relevant information from working memory but that older adults are not, younger adults showed no priming for the self-generated but disconfirmed words (stamp) coupled with above-baseline priming only for the to-beremembered target words (check); older adults showed priming for both the self-generated, disconfirmed words and the experimenter-provided, target words (Hartman \& Hasher, 1991). Thus, diminished inhibition appears to result in sustained activation of words (see also Zacks \& Hasher, 1994).

The central goal of the present work was to begin the process of determining circumstances that enable older adults (and by extension others having inefficient inhibition) to successfully abandon those ideas that are no longer relevant for a task. Using the Hartman and Hasher (1991) task, we explored two such circumstances: the provision (1) of elaborative semantic context and (2) of additional processing time. There is a rich literature in cognitive gerontology pointing to the beneficial role that additional context can play (in at least some circumstances) in supporting the performance of older adults across a range of tasks (see, e.g., Craik \& Jennings, 1992). Consistent with such findings is the recent report that additional, elaborative meaningful context enables older adults to reduce the range of ideas (sentence-ending words) that initially enter working memory (Stoltzfus, 1992). There is also, of course, a literature suggesting that slowing associated with aging may contribute to disruptions in processing (e.g., Cerella, 1985; Myerson, Hale, Wagstaff, Poon, \& Smith, 1990; Salthouse, 1988). To anticipate, we found that semantic context that explicates a change in meaning is effective in helping older adults to successfully delete no-longer-relevant thoughts from working memory. We also found that the simple provision of additional processing time following a change in meaning-without additional, elaborating semantic context-is not effective. The suggestion is that more information, not more time, is required for inefficient inhibitory mechanisms to clear the recent past from working memory.

\section{EXPERIMENT 1}

The Hartman and Hasher (1991) procedure demonstrates clearly the increased access that older adults have to no-longer-relevant words that were generated as endings to high-cloze sentences. Because of the task's importance as a measure of continued access to disconfirmed information, a straightforward replication of the original study was undertaken (see also Hartman \& Dusek, 1994, Experiment 1).

\footnotetext{
Method

Participants. Twenty-four younger adults ( $M=18.3$ years, range $=18-21$ years; 10 women and 14 men $)$ and 24 older adults $(M=69.1$ years, range $=60-74$ years; 8 women and 16 men $)$ participated. The participants were native English speakers. Al had normal or corrected-to-normal vision. The younger adults in Experiment 1 (and in Experiment 2) were university undergraduates who participated in experiments as one option for satisfying a course requirement. The older adults in Experiment I (and in
} 
Experiments 2 and 3 ) were volunteers from a registry maintained by the Duke University Center for the Study of Aging and Human Development. The older adults had completed more years of education ( $M=16.3$ years) than had the younger adults $(M=12.4$ years) $\left[F(1,46)=52.54, M S_{\mathrm{e}}=3.5\right]$. The older aduits also had a higher vocabulary score $(M=26.8, S D=6.3)$ than did the younger adults $(M=17.6, S D=6.3)\left[F(1,46)=25.17, M S_{\mathrm{e}}=39.57\right]$, as assessed by the Extended Range Vocabulary Test, Form 4 (ERVT; Educational Testing Service, 1976), a very difficult multiplechoice test.

Materials and Procedure. In the first phase of the study, the participants read a series of 28 high-cloze sentences, each of which was missing its final word. The task for the participant was to produce, aloud, a likely ending for each sentence. For critical sentences, the ending provided by the participant (and the one we anticipated) was replaced by a low-probable, but plausible, alternative as the targeted, to-be-remembered item. There were two sets of the 14 critical, high-cloze sentences and one set of 14 highcloze sentences that served as filler items. Each filler had its participant-generated ending confirmed. The filler sentences were given to all participants, with each of the two sets of critical sentences given to half of the participants in each age group. During the presentation phase, filler sentences were intermixed with experimental sentences, with the constraints that two fillers appeared at the beginning of the series and two appeared at the end and that no more than three experimental sentences (ones that had their participant-generated endings disconfirmed) appeared consecutively. In addition, two orders of presentation were constructed, and each order and sentence set combination was used equally often within each age group

Sentence frames were presented one at a time on a computer screen, each following a warning signal (a plus sign) that appeared centered on the screen for $750 \mathrm{msec}$. Each sentence frame was exposed until the participant generated a completion aloud. This response-initiated voice key generated a pause of $400 \mathrm{msec}$, after which the experimenter-provided final word appeared on the screen for $2,500 \mathrm{msec}$. For the 14 filler sentences, the experimenterprovided word was intended to match the participant-generated word, and it most often did so given the very high cloze value of those sentences. For critical sentences, the experimenter-provided word was the unexpected ending word. The participants' task was to remember the final word that appeared on the screen for a later, unspecified memory test. The materials were structured so that, for approximately half of the time, the word that appeared on the screen was the one the participant generated (filler sentences), and, for half of the time, it was a new word (critical sentences).

After finishing the sentence completion task, the participants performed an unrelated task for $5 \mathrm{~min}$ and then were asked to help norm materials for a new, unrelated experiment. Their task (an indirect memory test) was to read each of a series of 56 new sentence frames aloud. The participants were instructed to generate the first word that came to mind as a completion for the missing final word of each sentence. These were medium-cloze sentences, 28 of which were written (and normed) to converge about $50 \%$ of the time on an experimenter-provided target ending of a critical initial sentence and 28 of which were written (and normed) to converge on a self-generated but disconfirmed ending of a critical initial sentence. 'As a result, each of the 28 critical high-cloze sentences available for use in the first phase ( 14 of which were actually presented to a given participant) was associated with 2 medium-cloze sentences, and all 56 medium-cloze sentences were given to each participant under a self-paced procedure.

The critical question on the indirect test was the rate with which the participants completed moderate-cloze sentences with the target and disconfirmed words from the first part of the experiment, relative to the baseline rate of completion by controls who never saw the critical, first-phase sentences that suggested those words.
Thus, priming of self-generated, disconfirmed endings and of experimenter-provided target endings were the central effects of interest.

Because each participant saw only half of the critical high-cloze materials during Phase 1 and because all medium-cloze test phase sentences were presented to all participants in Phase 2, 28 of the medium-cloze sentences matched the two endings of the 14 critical sentences that half the participants had seen, whereas 28 matched the two endings of 14 critical sentences that the other participants had seen. This enabled us to counterbalance 28 high-cloze sentences across the experiment in such a way that, within each age group, the high-cloze sentences that served as the critical items (whose initial endings were rejected and replaced by to-beremembered targets) for half of the participants served as neverseen controls for the remaining participants.

The experiment proper began with two practice sentences. An informal quiz was used to ensure that each participant understood exactly what word was critical for the ensuing memory test (it was always the final word, and it was always presented on the screen). Following completion of the self-paced indirect memory test, the participants were questioned about their awareness of the relationship between the two main phases of the experiment. Two general questions were asked of all subjects: "What is your understanding of the purpose of this study so far?" and "When you were doing the sentence completions, did you think there was anything unusual about the sentences?" Further questions were more leading and were pursued only if a participant reported any awareness of the connection between the two tasks. After this interview, the participants were debriefed and were asked to complete the ERVT and a detailed health questionnaire. Four participants, 3 young adults and 1 older adult, were aware and were replaced by new participants from the same sources. This replacement procedure was also used in Experiments 2 and 3, with no more participants in those experiments reporting awareness than in Experiment 1.2

\section{Results and Discussion}

Study-trial sentence completions were examined to ensure that, for the critical experimental sentences, the subjects actually generated the expected, normatively highly probable endings. For younger adults, the mean proportion completed in this way was $96 \%$; for older adults, the mean was $95 \%$. These did not differ $(F<1)$. These completion rates were virtually identical for the two following experiments (range $=94 \%-97 \%$, again with no age differences). For all three experiments reported here, when the participants failed to produce the expected ending for a sentence during the first phase, performance on the two critical medium-cloze test-phase sentences was not scored.

There were three types of sentence frames present on the indirect memory test: (1) target word frames, which assessed the accessibility of the to-be-remembered words (e.g., check) that were provided by the experimenter, (2) disconfirmed word frames, which assessed the accessibility of expected words (e.g., stamp) initially generated by the participants, and (3) control word frames, which assessed the accessibility of the endings of critical sentences not actually presented to that participant (but presented to other participants). Completion rates for the three frame types, and priming scores measured as the difference in completion rates between each experimental condition and the control, are shown for each age group 
Table 1

\begin{tabular}{|c|c|c|c|c|c|c|c|c|c|c|}
\hline \multirow{3}{*}{$\begin{array}{c}\text { Adult } \\
\text { Participants }\end{array}$} & \multicolumn{6}{|c|}{ Sentence Frame Type } & \multicolumn{4}{|c|}{ Effect } \\
\hline & \multicolumn{2}{|c|}{ Target* } & \multicolumn{2}{|c|}{ Disconfirmed ${ }^{\dagger}$} & \multicolumn{2}{|c|}{ Control } & \multicolumn{2}{|c|}{ Target } & \multicolumn{2}{|c|}{ Disconfirmed } \\
\hline & $M$ & $S D$ & $M$ & $S D$ & $M$ & $S D$ & $\bar{M}$ & $S D$ & $M$ & $S D$ \\
\hline \multicolumn{11}{|c|}{ Experiment 1} \\
\hline Younger & 62.2 & 13.6 & 52.2 & 12.1 & 50.6 & 12.0 & 11.6 & 13.8 & 1.6 & 14.4 \\
\hline Older & 58.3 & 10.7 & 60.6 & 11.4 & 46.7 & 9.5 & 11.6 & 12.4 & 13.9 & 11.1 \\
\hline \multicolumn{11}{|c|}{ Experiment 2} \\
\hline Younger & 62.9 & 14.6 & 49.6 & 15.6 & 48.1 & 10.4 & 14.8 & 15.7 & 1.5 & 14.3 \\
\hline Older & 61.2 & 11.7 & 51.5 & 12.7 & 49.0 & 7.8 & 12.2 & 11.6 & 2.5 & 11.6 \\
\hline \multicolumn{11}{|c|}{ Experiment 3} \\
\hline Older & 58.7 & 13.9 & 59.0 & 12.3 & 51.4 & 8.1 & 7.3 & 15.5 & 7.6 & 14.0 \\
\hline
\end{tabular}

in Table 1. An initial analysis was done to determine whether there were age differences in completion rates for control sentences. There were not $[F(1,46)=1.52$, $\left.M S_{\mathrm{e}}=117.89, p>.22\right]$. Remaining analyses were then done on priming effect scores (also shown in Table 1).

A 2 (ages) $\times 2$ (sentence endings) mixed analysis of variance (ANOVA) was conducted on the priming scores. This confirmed what is apparent in the table: There was an overall age effect, with older adults showing greater priming than younger adults $\left[F(1,46)=4.18, M S_{\mathrm{e}}=\right.$ 205.11]. The overall difference between target and disconfirmed effects was not reliable $[F(1,46)=2.47, p=$ $.12]$; however, the interaction between age and priming condition was significant $\left[F(1,46)=6.46, M S_{\mathrm{e}}=132.6\right]$. Since older adults showed approximately equivalent priming for the two item types $(F<1)$, the interaction was clearly the result of younger adults' showing more priming for the target items than for the disconfirmed items $\left[F(1,23)=10.02, M S_{\mathrm{e}}=224.2\right]$. In fact, for young adults, the priming effect was reliably above the control baseline only for target items $\left[F(1,23)=16.93, M S_{\mathrm{e}}=103.7\right]$; the disconfirmed effect was not reliable $(F<1)$. For older adults, both target and disconfirmed endings were reliably different from controls $[F \mathrm{~s}(1,23)=20.94$ and $37.51, M S_{\mathrm{e}} \mathrm{s}=77.21$ and 61.7 , respectively].

These data are generally consistent with those originally reported by Hartman and Hasher (1991): Young adults have sustained access only to the experimenterprovided target ending of the initial high-cloze sentence. Older adults, by contrast, have access to both the target, or experimenter-provided final word, and their selfgenerated but disconfirmed ending word. The heightened access that older adults show to no-longer-relevant information is, we believe, the outcome of inefficient inhibitory mechanisms that fail to quickly suppress the activation of no-longer-relevant information, such as words and their meanings (see also Gernsbacher, 1990; Kintsch, 1993).
There is one difference between the present data and those reported by Hartman and Hasher (1991; see also Hartman \& Dusek, 1994): Here, the overall priming shown by older adults was actually higher than that shown by younger adults. This was the result of priming seen for disconfirmed words. These indirect memory findings, it should be noted, stand in sharp contrast to direct memory test findings reported by Hartman and Dusek (1994), who also used the Phase 1 disconfirmation procedure used here. They found better recall and recognition of target than of disconfirmed items, by both older and younger adults, and they also reported the overall age advantage that younger adults often have in laboratory memory tasks.

\section{EXPERIMENT 2}

Although the idea of inhibitory inefficiency has appeal at a theoretical level and, in fact, explains a range of findings in cognitive gerontology (see, e.g., Hasher \& Zacks, 1988; Stoltzfus et al., 1996; Zacks \& Hasher, 1994), it has problems at a pragmatic level in dealing with everyday behavior. This is because if inefficient inhibition continuously exposes working memory to overloadingparticularly, by nonrelevant information - the expected outcome would be disorganized behavior. That is, people with inefficient inhibitory mechanisms should have a great deal of difficulty in developing coherent streams of thought and action. Obviously, chaotic behavior does occur in older adults (as it does in adolescents and young adults), but it is clearly not common-particularly, for those community-dwelling adults who volunteer to participate in studies such as the present one.

What then spares older adults from the chaos that might be predicted on the basis of inefficient inhibition? Several factors have been suggested, including the fact that inhibitory mechanisms that serve spatial location information do not appear to be damaged with age (Carlson, 
Hasher, Connelly, \& Zacks, 1995; Connelly \& Hasher, 1993; see also Hasher \& Zacks, 1988). This enables information that occurs in predicted physical and imagined locations (see Radvansky, Zacks, \& Hasher, 1996) to be successfully ignored, thus reducing sources of distraction (see also Murphy \& McDowd, 1994). Another sparing factor has been reported in the emerging literature on chronocognition: Older adults have their optimal time of day in the morning, and performance (including inhibitory control) is often better, if not quite up to the level of younger adults, when older adults are tested at their optimal times (May \& Hasher, in press; May, Hasher, \& Stoltzfus, 1993). An additional sparing factor, and the one explored here, might well be tied to the fact that information is often embedded in a rich and meaningfully appropriate elaborative semantic context.

For example, there is evidence that the size of the memory deficits shown by older adults across most explicit memory tasks can sometimes be reduced when precise, relative to imprecise, elaborators are available (e.g., Hashtroudi, Parker, Luis, \& Reisen, 1989; Rankin \& Collins, 1986; see also Craik \& Jennings, 1992). There is also evidence that context aids perceptual recognition of both visually and auditorially presented words (Cohen \& Faulkner, 1983). Stoltzfus (1992) has recently shown that the provision of a meaningfully appropriate, enriched semantic context enables older adults to narrow the range of ideas that initially enter working memory. In one study, she found that for a high-cloze sentence, such as "The landlord was afraid of a strike by the

older adults had speeded access to at least two different endings (tenants and residents) when only one (tenants) was normatively highly predicted. Under these same circumstances, younger adults had access only to the highly expected ending (tenants). In an additional experiment, participants read each of the critical, high-cloze sentences but only after they read a context-setting sentence (e.g., "The living conditions in the apartment building had deteriorated seriously. The landlord was afraid of a strike by the "). In this instance, both older and younger adults showed speeded access only to highly predicted endings, suggesting that relevant semantic context may offer the kind of contextual support that Craik (1986) has argued particularly boosts the cognitive efficiency of older adults. In any event, the Stoltzfus data suggest that relevant semantic context can aid impaired inhibitory mechanisms that control access to working memory.

Here, we considered the possibility that the active inhibitory process of abandoning no-longer-relevant information from working memory can also be aided by relevant semantic context. We used the garden-path disconfirmation procedure and materials of Experiment 1, but, in Experiment 2, each sentence was followed by an additional, elaborating sentence. For example, the critical sentence, "He mailed the letter without a ___ " (to which most participants generated stamp and for which the experimenter-provided target word was check), was followed by an elaborating sentence, "He was expecting the money." The elaborating sentences were designed to explain the unexpected ending of the preceding sentence. The question in Experiment 2 was whether or not the provision of an elaborating sentence would result in older adults" successful elimination of no-longer-relevant words (the self-generated endings to high-cloze sentence frames).

\section{Method}

Participants. The participants were sampled from the same groups as in Experiment 1 and now included 24 younger adults ( 15 women and 9 men) and 24 older adults ( 13 women and $11 \mathrm{men}$ ), none of whom had served in Experiment 1 . The mean age of the younger adults was 18.8 years (range $=17-22$ years). They had completed a mean of 12.5 years $(S D=0.88)$ of education. The mean age of the older adult participants was 69.6 years (range $=$ 63-79 years). They had completed a mean of 15.4 years $(S D=2.75$ ) of education, which was reliably more than that for the younger adults $\left[F(1,46)=23.13, M S_{\mathrm{e}}=4.17\right]$. The older adults had higher vocabulary scores $(M=34.5, S D=8.0)$ than did the younger adults $(M=29.0, S D=7.4)\left[F(1,46)=6.12, M S_{c}=59.32\right]$.

Materials and Procedure. Twenty-eight sentence pairs served as critical experimental materials. Each set consisted of 2 sentences, with the first one missing a final word and the second one elaborating on the meaning of an unexpected ending that was provided for the first one. The first sentence in each set was the same as the critical sentences from Experiment 1 . As before, the initial set of 28 pairs of sentences was divided into two subsets of 14 sentence pairs, with each set used for half of the subjects in each age group. The participants received the identical set of 14 filler sentences from Experiment 1, together with a second sentence that elaborated upon the expected ending word. No other aspects of the materials were changed from Experiment 1

The procedures were identical to those of Experiment 1 except that, following the exposure (for $2,500 \mathrm{msec}$ ) of the target ending, the elaborating sentence appeared on the screen along with the first sentence for an additional $3,000 \mathrm{msec}$, after which the sequence began again until the last of the 28 pairs ( 14 critical, 14 fillers) was presented. As before, the experiment began with two practice sentence sets, both with unexpected endings, and an informal quiz to ensure that each participant understood exactly which word was critical for the ensuing memory test.

\section{Results and Discussion}

The completion rates for target, disconfirmed, and control items are shown in Table 1. As before, and again consistent with norms, the expected completion rate of words for sentences that had never been seen in the context of the study hovered at around 50\%. Because there were again no age differences in completion rates in the control condition $(p>.10)$, further analyses were conducted using the repetition priming scores for the target and disconfirmed conditions.

A two-way ANOVA was conducted with age (young vs old) as a between-subjects variable and condition (target vs. disconfirmed) as a within-subjects variable. The total amount of repetition priming did not differ between younger and older adults $(F<1)$; in contrast to the results of Experiment 1 and to earlier findings, there was no interaction between age and priming condition $(F<1)$. In Experiment 2, the older adults showed the same patterns of repetition priming as did the younger adults. As can clearly be seen. there was greater repetition priming for 
target words than for disconfirmed words (overall, $13.5 \%$ vs. $2.0 \%)\left[F(1,46)=19.2, M S_{\mathrm{e}}=166.3\right]$. Priming for the targets, but not the disconfirmed items, was reliably greater than zero $\left[F(1,46)=46.6, M S_{\mathrm{e}}=94.5\right.$, and $F(1,46)=1.2, M S_{\mathrm{e}}=83.2$, respectively, $\left.p>.25\right]$. Thus, both younger and older adults showed repetition priming for the target words but not for the disconfirmed words. The provision of elaborating, explanatory context apparently does enable older adults to narrow processing to an experimenter-provided, unexpected word, even in the face of having available a more plausible, self-generated word. The results of Experiment 2 are consistent with the findings of Stoltzfus (1992) in showing that, under some circumstances, older adults can narrow the maintenance of interpretations to the one that is consistent with the final interpretation of a message.

The garden-path nature of the sentences (in which a highly probable, participant-generated word is replaced by a less probable, experimenter-provided one) requires the use of materials that are not counterbalanced (i.e., the words that serve in the disconfirmed condition are different from those in the target condition). Therefore, the findings from Experiments 1 and 2, taken together, disconfirmed one interpretation (see Hartman, 1995) of the findings of Experiment 1 -that is, that there is a materials $\times$ age interaction such that for young adults those items that served as targets are more memorable than those that served as the disconfirmed items, while for older adults (with their greater word knowledge), the two sets of items were equally memorable. Since the provision of an elaborating context for the new target word makes that item equally memorable for older adults (and the disconfirmed word equally unmemorable), such an interpretation of Experiment l's findings is untenable.

In Experiment 2, two factors might have contributed to the effective narrowing of activation of meaning shown here by older adults: (1) the additional sentence, which provided an integrating explanation for the unexpected ending of the critical first sentence, or (2) the additional $3,000 \mathrm{msec}$ of processing time available to the participants following the provision of the unexpected, to-beremembered ending. This was the time provided for the participants to read the elaborating, second sentence and to consider the two sentences together. Thus, the presence of the second sentence in Experiment 2 created an intersentence interval that did not exist in Experiment I. Since many behaviors are slowed with age (e.g., Salthouse, 1988), it is conceivable that the additional time alonenot the meaningful context- - was responsible for the reduced activation that the older adults showed for the initially self-generated ending. This issue was addressed in Experiment 3.

\section{EXPERIMENT 3}

The central question here was whether the priming effects seen for the older adults in Experiment 2 were the result of the additional interpretive context provided by the elaborating sentence that followed the critical, initial sentence, or whether the narrowed meaning access was the result of the additional time that was available to interpret the initial sentence, time provided during the reading of the second, elaborating sentence. To determine whether time alone allows older adults to successfully abandon a disconfirmed interpretation, a replication of Experiment 2 was conducted, with two changes. First, the elaborating sentence used in Experiment 2 was eliminated, leaving only an unfilled interval of $3,000 \mathrm{msec}$ between the end of one sentence and the onset of the warning signal for the next sentence. Second, only older adults were tested. If time alone is sufficient to eliminate activation to no-longer-relevant interpretations, the meaning access pattern of older adults in this experiment should replicate that seen in Experiment 2 for both older and younger adults; only the experimenter-provided target ending should be accessible. If, however, elaborative information is required for suppression of no-longerrelevant interpretations to occur, the priming pattern produced by older adults who are given more time should look like those reported in Experiment 1 , in which older adults showed sustained activation for their self-generated, expected endings and for the experimenter-provided, unexpected endings. On the basis of findings reported by Stoltzfus (1992), our expectation was that the latter finding would obtain.

\section{Method}

Participants. The participants were 24 healthy older adults ( 16 women and $8 \mathrm{men}$ ) who had not participated in Experiments 1 and 2. The mean age of these participants was 69.3 years ( range $=$ 62-77 years, $S D=3.9$ ). They had completed an average of 16.2 years $(S D=2.9)$ of education. Neither the ages nor the years of education of the older adults in Experiment 3 differed from those of the older adults in Experiment $2(F<1.02)$. Their mean vocabulary score $(E R V T)$ was $35.5(S D=8.7)$, a score that did not differ from that obtained by the subjects who participated in Experiment $2(F<1)$.

Materials and Procedure. The materials and procedures of Experiment 3 were identical to those of Experiment 2, with the critical exception of the omission of the elaborating sentence used during the study phase in the previous experiment. Instead, after the provision of the experimenter-provided ending, the screen remained blank for an additional $3,000 \mathrm{msec}$ (the same amount of processing time allowed for reading the second sentence in Experiment 2 ). The participants were instructed here, as before, that the task was to remember the last word provided for each sentence.

\section{Results and Discussion}

An inspection of the data (see Table 1) shows a very different pattern from that seen in Experiment 2: Here, older adults showed equivalent priming for both target and disconfirmed endings ( $7.3 \%$ and $7.6 \%$, respectively) $(F<1)$. Both the target priming effect $[F(1,23)=5.3$, $\left.M S_{\mathrm{e}}=120.75\right]$ and the disconfirmed effect $[F(1,23)=$ $\left.7.1, M S_{\mathrm{e}}=97.85\right]$, were significantly different from zero.

To assess the patterns of priming effects that older adults show when additional context plus processing time are provided (in Experiment 2) versus when only additional time is provided (Experiment 3), a two-way 
mixed ANOVA was conducted for the older adult participants with study condition (or Experiment 2 vs. Experiment 3 ) as a between-subjects variable and item type (target vs. disconfirmed) as a within-subjects variable. The context condition $\times$ item type interaction was at a borderline value for significance $[F(1,46)=3.65, p=.06]$, suggesting different patterns of priming in the two experiments. Equivalent target and disconfirmed effects are seen when additional time only is provided (Experiment 3 ). There is reliable priming only for the target, but not the disconfirmed item, when additional context is provided (Experiment 2).

The presence of reliable and equivalent priming for targets and disconfirmed items seen here is, in pattern, similar to the data for older adults from Experiment 1 and also to those reported initially by Hartman and Hasher (1991; see also Hartman \& Dusek, 1994): When the garden path was reversed, older adults, unlike younger adults, maintained access to both sentence-ending words (the self-generated and experimenter-provided ones) of many sentences. The results of Experiment 3 clearly demonstrate that additional time after the provision of an unexpected ending does not alter what words older adults continue to maintain access to across time. This suggests that, for older adults, reversal of a garden path (or recognizing the existence of a misunderstanding) does not automatically elicit a process that eliminates no-longer-relevant or incorrect information from consideration--a suggestion consistent with the earlier findings of Hamm and Hasher (1992), who used both different materials (paragraphs) and a different testing procedure (speeded decisions) from those in the present instance. Of particular importance is the conclusion that slowing is not a viable explanation of the activation patterns shown by older adults when they have been led down an interpretive garden path. Nor, given the high verbal skills and levels of education of the present groups of older adults, can one attribute the pattern of findings of sustained activation seen here to a misunderstanding or to a lack of relevant interpretive knowledge.

The present data suggest that, although older adults can acquire a new interpretation for the ending of a sentence, they neither (1) suppress a no-longer-relevant ending nor (2) spontaneously generate elaborative interpretations that would, if otherwise provided, enable them to abandon a no-longer-correct interpretation (see Craik \& Jennings, 1992). For older adults (and possibly for others with inefficient inhibitory systems), the successful elimination of no-longer-relevant words or interpretations requires additional information that elaborates and specifies the meaning of the new interpretation. Elsewhere, it has been argued that the establishment of meaning from discourse requires elaboration and suppression processes (see, e.g., Kintsch, 1993) that are believed to be well developed in skilled readers (Gernsbacher, 1990). Since the older adults in the present experiments surely have high levels of verbal ability, the extension of such models to aging requires careful consideration of possible age differences in such processes. At a pragmatic level, the present data suggest that discourse targeted to even highly verbal older adults needs to take into account differences in spontaneous elaboration and suppression processes.

\section{GENERAL DISCUSSION}

Three experiments used a garden-path methodology in which an initial, high-probable ending of critical sentences was replaced by a low-probable, but plausible, tobe-remembered word. The empirical question was whether older adults can abandon their initially generated, but nolonger-relevant word, to focus on a new, relevant one. This question is an important one in ordinary language processing because the present garden-path situation models many everyday occurrences in which a once-relevant idea (or topic, character, or setting) is replaced by a new one. We (Hasher \& Zacks, 1988) and others (e.g., Gernsbacher, 1990; Kintsch, 1993) have assumed that the deletion of a once-relevant interpretation from working memory requires the operation of active inhibitory processes. Since an increasingly extensive line of work suggests that older adults have deficient inhibitory attentional mechanisms (e.g., Arbuckle \& Gold, 1993; Hasher, 1994; Lindfield, Wingfield, \& Bowles, 1994; McDowd et al., 1995; Stoltzfus et al., 1996; Zacks, 1995; Zacks \& Hasher, 1994; but see, e.g., Stoltzfus et al., 1996, for criticisms and limitations of aging claims), it is reasonable to anticipate that situations in which there is a change in focus-or in which errors of interpretation need to be deleted-will present particular problems for older adults.

Using a sentence-completion task as an implicit test, the present experiments assessed the degree to which the relevant, to-be-remembered target items and the no-longerrelevant, self-generated items were accessible sometime after a switch of interpretation occurred. The results of Experiment 1 replicated findings initially reported by Hartman and Hasher (1991): Under these testing circumstances, younger adults showed access only to the target words; the no-longer-relevant words returned to their baseline of accessibility. By contrast, older adults showed access to two ending words, both the previously correct one and the currently held one. Experiment 2 demonstrated one critical circumstance under which older adults can succeed in eliminating no-longer-relevant information from working memory: when an elaborating interpretation is provided for an unexpected or unlikely ending of a sentence. In this case, older adults look like younger adults; their originally generated sentence-ending words returned to baseline accessibility levels and only the final, experimenter-provided ending was still accessible. Experiment 3 demonstrated that the narrowing of activation that additional elaborating context enabled for older adults in Experiment 2 cannot be explained by the additional time that was needed to read that elaborating sentence: An equivalent unfilled interval did not permit narrowing of activation to a single target interpretation.

Other evidence suggests that additional information alone, just like additional time alone, does not enable older adults to narrow their interpretation to a final, correct 
one. This evidence comes from a study that used gardenpath paragraphs to mislead older and younger readers to an initial inference that subsequently turned out to be incorrect (Hamm \& Hasher, 1992). In those materials, two or three sentences followed the point in the passage at which the garden path was reversed. However, those additional sentences did not elaborate on the new inference; instead, they generally continued the story, without specific reference to the new interpretation. There, older adults maintained two inferences: the original, misguided, but highly probable one, and the final correct, but less likely one. Thus, together, these two sets of studies suggest that neither filled nor unfilled intervals allow older adults to narrow activation to a single interpretation. Instead, on the basis of the results of Experiment 2, it appears that specifically relevant, elaborative context is required to abandon an activated interpretation.

It is possible that the interpretive behavior of older adults in these garden-path studies represents a style that attempts an inclusive interpretation. That is, when a garden path is traversed, young adults' goal is to have a single, exclusive interpretation available, whereas older adults' goal is to have an integrative one that encompasses multiple possible interpretations (see Boswell, 1979). ${ }^{3}$ If so, perhaps the inclusive interpretational attempts are abandoned only when additional information clearly dictates an exclusive interpretation. Such an argument is similar to the suggestion that older adults have difficulty selecting an interpretation in the face of a potential competitor, as is the case for garden-path materials (e.g., Hartman, 1995). Alternatively, it is possible that, like younger less-skilled readers, older skilled readers fail to spontaneously generate "topic-level" inferences or situation models that explain and link more local inferences and propositions that are themselves largely textdriven (see, e.g., Kintsch, 1993; Long, Oppy, \& Seely, 1994). It is conceivable that these high-level interpretations trigger the inhibition of any lower level interpretations that are inconsistent. Furthermore, it is possible that the generation of a larger number of lower level interpretations slows or even makes difficult the generation of higher level, integrative interpretations. These speculations, in turn, suggest that there may be circumstances in which explicit text-level linkages must be provided for older adults.

What general cognitive mechanisms might account for the present data? Capacity-type arguments (see, e.g., Hasher \& Zacks, 1979; Just \& Carpenter, 1992; Light, 1991), which assume that older adults have less capacity than do younger adults, would suggest that narrowing of meaning is a more demanding process than is establishing a broad interpretation. Such an explanation of the present data seems unsuitable, however. Consider that successful narrowing of activated meanings (which, on such an account, requires capacity) occurs under the load conditions of Experiment 2 (the processing of an extra sentence), but not under the no-load conditions of Experiment 3 (unfilled time). This is the reverse of findings expected from a capacity perspective. Of course, one would also predict that limited capacity would lead to fewer, not more, interpretations being available to comprehenders in the first instance. Older adults do have more interpretations per sentence available than younger adults do. Slowing, too, seems an inadequate interpretation: More time (without explicit elaboration) clearly does not allow for older adults to narrow activation to a single meaning.

One alternative interpretation is tied to deficient inhibitory mechanisms, which, in turn, permit sustained activation of the most recent information, even when that information is no longer correct. Sustained activation, in turn, may limit or preclude the self-initiated activities that effective text-level interpretations and remembering (e.g., Kintsch, 1993) both require. Evidence consistent with the inhibitory view comes from recent work with young adults who were tested in the task used in Experiment 1 at their optimal versus their nonoptimal time of day (May \& Hasher, in press). At their nonoptimal time, young adults showed priming to both disconfirmed and target endings. (This is, of course, the pattern shown by older adults in Experiments 1 and 3 and, as we now know, the pattern seen even when older adults are tested only at their optimal times. ${ }^{4}$ ) However, at their optimal time, young adults showed access only to the target ending; access to the disconfirmed ending was actually below baseline- - that is, the disconfirmed words were actually less accessible than were never-presented control words. It is difficult to account for such suppression-type effects without relying on an inhibitory attentional mechanism that functions to clear working memory so as to orient the comprehender away from no-longer-relevant topics. Note that, in May and Hasher's (in press) study, older adults who were tested at their nonoptimal time showed memory only for the disconfirmed item; they were totally unable to suppress their self-generated ending. This is a finding consistent with a deficient-inhibition viewpoint.

Thus, taken together, these findings (from the present experiments and the work of Hamm \& Hasher, 1992, and from May \& Hasher, in press) would suggest that older adults have deficient inhibition that makes it difficult to clear working memory of no-longer-relevant information. ${ }^{5}$ Older adults are able to compensate, to some degree, for inefficient inhibitory attentional mechanisms when explicitly elaborating information provides the text-level interpretative materials that they seem not to produce spontaneously in the face of sustained activation of alternative meanings. Finally, the present work also strengthens arguments made in some text-comprehension frameworks (e.g., Gernsbacher, 1990; Kintsch, 1993) and in attentional-control frameworks (e.g., Hasher \& Zacks, 1988; Hasher, Zacks, \& May, in press; Stoltzfus et al., 1996) that inhibitory mechanisms are critical for the establishment of cohesion in thought and action.

\section{REFERENCES}

Arbuckle. T. Y., \& Gold, D. P. (1993). Aging, inhibition, and verbosity. Joturnal of Gerontology": Psichological Sciences, 48, P225P232. 
BEECH, A., \& CLARIDGE, G. (1987). Individual differences in negative priming: Relations with schizotypal personality traits. British Journal of Psychology, 78, 349-356.

BosWeLL, D. A. (1979). Metaphoric processing in the mature years. Human Development, 22, 373-384

Carlson, M. C., Hasher, L., Connelly, S. L., \& Zacks, R. T. (1995). Aging, distraction and the benefits of predictable location. Psychology \& Aging, 10, 427-436.

Cerella, J. (1985). Information processing rates in the elderly. PSychological Bulletin, 98, 67-83.

COHEN, G., \& Faulkner, D. (1983). Word recognition: Age differences in contextual facilitation effects. British Journal of Psychology, 74, 239-25l.

CONNelly, S. L., \& Hasher, L. (1993). Aging and the inhibition of spatial location. Journal of Experimental Psychology: Human Perception \& Performance, 19, 553-541.

Craik, F. I. M. (1986). A functional account of age differences in memory. In F. Klix \& H. Hagendorf (Eds.), Human memory and cognitive capabilities, mechanisms and performances (pp. 409422). Amsterdam: Elsevier, North-Holland.

Craik, F. I. M., \& Jennings, J, M. (1992). Human memory. In F. I. M. Craik \& T. A. Salthouse (Eds.), The handbook of aging and cognition (pp. 51-110). Hillsdale, NJ: Erlbaum.

DEMPSTER, F. N. (1992). The rise and fall of the inhibitory mechanisms: Toward a unified theory of cognitive development and aging. Developmental Review, 12, 45-75.

Educational Testing Service (1976). Kit of factor-referenced cognitive tests. Princeton, $\mathrm{NJ}$ : Author

Ferraro, F. R., Johnson, K., \& Wonderlich, S. (1995, May). Negative priming deficits in individuals at risk for eating disorders. Paper presented at the annual meeting of the Midwestern Psychological Association, Chicago.

Frith, C. D. (1979). Consciousness, information processing, and schizophrenia. British Journal of Psychology, 134, 225-235.

GERNSBACHER, M. A. (1990). Language comprehension as structure building. Hillsdale, NJ: Erlbaum

HAMM, V. P., \& HASHER, L. (1992). Age and the availability of inferences. Psychology \& Aging, 7, 56-64.

HARNISHFEGER, K. K., \& BJORKLUND, D. F. (1993). The ontogeny of inhibitory mechanisms: A renewed approach to cognitive development. In M. L. Howe \& R. Pasnak (Eds), Emerging themes in cognitive development (pp. 28-49). New York: Springer-Verlag.

HARTMAN, M. (1995). Aging and interference: Evidence from indirect memory tests. Psychology \& Aging, 4, 659-669.

HaRTMAN, M., \& DUSEK, J. (1994). Direct and indirect memory tests: What they reveal about age differences in interference. Aging \& Cognition, 1, 292-309.

HaRTMAN, M., \& HASHER, L. (1991). Aging and suppression: Memory for previously relevant information. Psychology \& Aging, 6, 587-594.

HASHER, L. (1994, July). Inhibition and cognition. Paper presented at the annual meeting of the American Psychological Society, Washington, DC.

Hasher, L., \& ZACKs, R. T. (1979). Automatic and effortful processes in memory. Journal of Experimental Psychology: General. 108, 356-388.

HASHER, L., \& ZACKS, R. T. (1988). Working memory, comprehension. and aging: A review and a new view. In G. H. Bower (Ed.), The psychology of learning and motivation (Vol. 22. pp. 193-325). San Diego: Academic Press.

HASHER, L., ZACKS, R. T., \& MAY, C. P. (in press). Inhibitory control, circadian arousal, and age. In D. Gopher \& A. Koriat (Eds.), Attention and performance $X V I I$ : Cognitive regulation of performance. Interaction of theory and application. Cambridge, MA: MIT Press.

Hashtroud, S., Parker, E. S., Luis, J. D., \& Reisen, C. A. (1989). Generation and elaboration in older adults. Experimental Aging Reseurch, 15, 73-78.

Just, M. A., \& CARPENTER, P. A. ( 1992 ). A capacity theory of comprehension. Psychological Review, 99, 122-149.

Kemper, S., Rash, S. R., Kynette, D., \& Norman, S. (1990). Telling stories: The structure of adults' narratives. European Journal of Cognitive Psichologr, 2, 205-228.
KINTSCH, W. (1993). Information accretion and reduction in test processing: Inferences. Discourse Processes, 16, 193-202

LAVER, G. D. (1992, April). A speed-accuracy analysis of semantic priming effects in young and older adults. Paper presented at the Cognitive Aging Conference, Atlanta

LAVER, G. D. (1994, April). Vocabulary and short-term memory span as mediators of the speed of semantic priming in young and older adults. Paper presented at the Cognitive Aging Conference, Atlanta.

LiGH T, L. L. (1991). Memory and aging: From hypotheses in search of data. Annual Review of Psychology, 42, 333-376.

LindFiELd, K. C., WingField, A., \& Bowles, N. L. (1994, April). Age differences in the Bruner-Potter interference effect: Evidence for the inhibition-deficit view. Paper presented at the Cognitive Aging Conference, Atlanta.

LiNviLLE, P. W. (1996). Attention inhibition: Does it underlie ruminative thought? In R. S. Wyer (Ed.), Advances in social cognition: Ruminative thoughts (Vol. 9, pp. 121-133). Mahwah, NJ: Erlbaum.

LONG, D. L., Oppy, B. J., \& SEely, M. R. (1994). Individual differences in the time course of inferential processing. Journal of Experimental Psychology: Learning, Memory, \& Cognition, 20, 1456-1470.

MAY, C. P. (1995). Circadian arousal and cognition: Evidence for synchrony effects in inhibition. Unpublished doctoral dissertation. Duke University.

MAY, C. P., \& HASHER, L. (in press). Synchrony effects in inhibitory control over thought and action. Journal of Experimental Psychology: Human Perception \& Performance.

May, C. P., Hasher, L., \& Stoltzfus, E. R. (1993). Optimal time of day and the magnitude of age differences in memory. Psychological Science, 4, 326-330

McDowd, J. M., Oseas-Kreger, D. M., \& Filion, D. L. (1995). Inhibitory processes in cognition and aging. In F. N. Dempster \& C. J. Brainerd (Eds.), Interference and inhibition in cognition (pp. 363 400). San Diego: Academic Press.

Mergler, N., FAust, M., \& Goldstein, M. (1985). Storytelling as an age-dependent skill. Internationai Journal of Aging \& Human Development, 20, 205-228

MurPhy. D. R., \& MCDowD, J. M. (1994, April). The influence of selective attention on speech processing in noise: The effect of con textually related and unrelated noise. Paper presented at the Cognitive Aging Conference, Atlanta.

Myerson, J., Hale, S., Wagstaff, D., Poon, L. W., \& Smith, G. A (1990). The information-loss model: A mathematical theory of agerelated cognitive slowing. Psvchological Review, 97, 475-487.

Pennington, B. F. (1994). The working memory function of the prefrontal cortices: Implications for developmental and individual differences in cognition. In M. Haith, J. B. Benson, R. J. Roberts, \& B. F. Pennington (Eds.), Future oriented processes in development (pp. 243-289). Chicago: University of Chicago Press

Pennington, B. F., Groisser, D., \& Welsh, M. C. (1993). Contrasting cognitive deficits in attention deficit hyperactivity disorder versus reading disability. Developmental Psvchologv, 29, 511-523.

Pratt, M. W., \& Robbins, S. L. (1991). That's the way it was: Age differences in the structure and quality of adult's personal narratives Discourse Processes, 14, 73-85.

Radvansky, G. A., ZaCks, R. T., \& HaSher, L. (1996). Fact retrieval in younger and older adults: The role of mental models. Psycholog! \& Aging, 11, 258-271.

Rankin, J. L., \& Collins, M. (1986). Adult age differences in memory elaboration. Journal of Gerontologi, 40, 451-458

Salthouse, T. A. (1988). Effects of aging on verbal abilities: Examination of the psychometric literature. In L. L. Light \& D. M. Burke (Eds.), Language, memory, and aging (pp. 17-35). Cambridge: Cambridge University Press.

Shimamura, A. (1995). Memory and frontal lobe function. In M. S Gazzaniga (Ed.). The congitive netwrosciences (pp. 803-813). Cambridge, MA: MIT Press.

Stine, E. A. L., Ruh, J. L., \& Hindman, J. (1994, April). The etfects of age and working memory capacity on reading time allocation and comprehension of ambiguous text. Paper presented at the Cognitive Aging Conference, Atlanta.

STOLT 2FLS. E. R. (1992). Aging and breadth of availability during 
language processing. Unpublished doctoral dissertation, Duke University.

Stoltzfus, E. R., Hasher, L., \& Zacks, R. T. (1996). Working memory and aging: Current status of the inhibitory view. In J. T. E. Richardson, R. W. Engle, L. Hasher, R. H. Logie, E. R. Stoltzfus, \& R. T. Zacks (Eds.), Working memory and human cognition (pp. 6688). Oxford: Oxford University Press.

Stoltzfus, E. R., Hasher, L., Zacks, R. T., Ulivi, M. S., \& GoldSTEIN, D. (1993). Investigations of inhibition and interference in younger and older adults. Journal of Gerontology: Psychological Sciences, 48, P179-P188.

ZACKS, R. T. (1995, May). Attending and remembering. Paper presented at the annual meeting of the Midwestern Psychological Association, Chicago.

ZACKS, R. T., \& HASHER, L. ( 1988), Capacity theory and the processing of inferences. In L. Light \& D. Burke (Eds.), Language, memory, and aging (pp. 154-170). New York: Cambridge University Press.

ZACKS, R. T., \& HASHER, L. (1994). Directed ignoring: Inhibitory regulation of working memory. In D. Dagenbach \& T. H. Carr (Eds.), Inhibitory processes in attention, memory, and language (pp. 241264). San Diego: Academic Press.

ZaCKs, R. T., \& HASHeR, L. (1996). Attentional inhibition in memory and language processing. Manuscript submitted for publication.

\section{NOTES}

1. These norms were reconfirmed within the context of the present experiment, with $49.8 \%$ of the participants generating the "selfgenerated" word and $51.0 \%$ generating the "target" word to the medium- cloze sentences, when the critical initial sentence was not exposed during Phase 1 of the task.

2. No comparisons were possible between aware and unaware participants, because so few fell into the former category.

3. It is possible (as Stoltzfus et al., 1996, have argued) that this tendency toward inclusion is part of the process that results in more interesting stories being told by older adults than by younger adults (Kemper, Rash, Kynette, \& Norman, 1990; Mergler, Faust, \& Goldstein, 1985; Pratt \& Robbins, 1991).

4. Explicit records of time of testing, along with an assessment of circadian rhythms, were not kept for the present investigation. However, since 1993, this laboratory has tended to test older adults only in morning hours, at least whenever possible (see May et al., 1993).

5. Enriched (or cluttered) working memory, we have argued elsewhere (Hasher \& Zacks, 1988), creates comprehension problems via the larger memory "fans" that are created during encoding, which, in turn, can result in slowed and inaccurate retrieval of target items in the cluttered fans (e.g., Zacks \& Hasher, 1994). These retrieval errors, then, result in failed on-line attempts to create the critical links between separate ideas that enable understanding and that aid the formation of coherent representations of discourse. It is worth noting that the enrichment of working memory during encoding (or the failure to narrow activation to just that information most relevant to ongoing interpretation) may also result in the more highly regarded stories that older adults tell relative to those told by younger adults (see Pratt \& Robbins, 1991).

(Manuscript received August 28, 1995; revision accepted for publication February $27,1996$.

\section{Nominations for the Editorship of Perception \& Psychophysics}

Nominations are solicited for the editorship of Perception \& Psychophysics. The term of the present editor, Myron Braunstein, expires at the end of 1998. The new editor will begin an official 4-or 5-year term on January 1, 1999, and will begin to receive manuscripts early in January 1998. The Publications Committee of the Psychonomic Society expects to appoint the new editor by November 1997.

Nominations (including self-nominations) should be submitted by August 1, 1997, to:

Stephen Palmer

Chair, Perception \& Psychophysics Search Committee

Department of Psychology

University of California

Berkeley, CA $94720-1650$ 